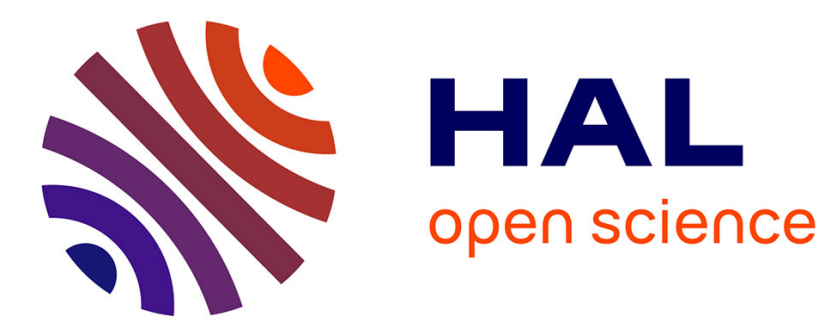

\title{
Contribution à l'étude écophysiologique de la phase semis-levée du ray-grass d'Italie (Lolium multi- florum Lam.) I. - Etude en conditions contrôlées de l'influence du facteur thermique
}

Jean-Claude Simon

\section{To cite this version:}

Jean-Claude Simon. Contribution à l'étude écophysiologique de la phase semis-levée du ray-grass d'Italie (Lolium multi- florum Lam.) I. - Etude en conditions contrôlées de l'influence du facteur thermique. Agronomie, 1981, 1 (5), pp.339-344. hal-00884263

\section{HAL Id: hal-00884263 \\ https://hal.science/hal-00884263}

Submitted on 1 Jan 1981

HAL is a multi-disciplinary open access archive for the deposit and dissemination of scientific research documents, whether they are published or not. The documents may come from teaching and research institutions in France or abroad, or from public or private research centers.
L'archive ouverte pluridisciplinaire HAL, est destinée au dépôt et à la diffusion de documents scientifiques de niveau recherche, publiés ou non, émanant des établissements d'enseignement et de recherche français ou étrangers, des laboratoires publics ou privés. 


\title{
Contribution à l'étude écophysiologique de la phase semis-levée du ray-grass d'Italie (Lolium multi- florum Lam.) I. - Etude en conditions contrôlées de l'influence du facteur thermique
}

Jean-Claude SIMON

I.N.R.A., Station d'Agronomie, 4, rue de Stang Vihan, F 29000 Quimper.

\begin{abstract}
RÉSUMÉ
Cette étude sur le ray-grass d'Italie, menée en conditions contrôlées, met en évidence le rôle du facteur température sur les différentes phases de croissance et de développement qui conduisent à la levée. La vitesse d'imbibition des semences augmente avec la température ; la capacité d'absorption d'eau par la semence n'est pas modifiée par ce facteur. $0^{\circ} \mathrm{C}$ est le seuil inférieur pour la germination et $1{ }^{\circ} \mathrm{C}$ le seuil pour la croissance de la radicule et du coléoptile. Au-dessus, la vitesse de croissance et de développement augmente avec la température jusqu'à un optimum thermique compris entre 20 et $25^{\circ} \mathrm{C}$. Au-delà, cette vitesse diminue rapidement pour s'annuler à $29^{\circ} \mathrm{C}$ (croissance des organes) et $32^{\circ} \mathrm{C}$ (germination). Entre le seuil thermique inférieur et l'optimum, vitesse de développement et température sont étroitement liées par une relation de type linéaire.

\section{Ecophysiologie}

Germination

Température
\end{abstract}

\begin{abstract}
SUMMARY
Contribution to the ecophysiological study of Italian rye-grass from sowing to emergence I. - Temperature influence in controlled conditions

Ecophysiology Italian rye-grass Emergence

Germination

Temperature

This study on Italian rye-grass made in controlled conditions stressed the effect of temperature on the different growth and development stages which lead to emergence.

The speed of the seed imbibition increased with temperature; the seed ability for absorbing water is not affected by temperature. $\mathrm{O}^{\circ} \mathrm{C}$ is the inferior threshold for germination and $1{ }^{\circ} \mathrm{C}$ the threshold for the radicle root and shoot growth. Above it the growth and development speed increased with temperature up to an optimum temperature from 20 to $25^{\circ} \mathrm{C}$. Beyond this temperature this speed decreased rapidly to reduce to zero at $29^{\circ} \mathrm{C}$ (organ growth) and $32{ }^{\circ} \mathrm{C}$ (germination). Between the temperature threshold and the optimum, development speed and temperature are closely related by a relation of linear type.
\end{abstract}

\section{I. - INTRODUCTION}

Les rapports entre facteurs du milieu et croissance et développement du ray-grass d'Italie sont pratiquement méconnus si l'on se réfère à une bibliographie fort réduite. Cette lacune est d'autant plus grave que cette plante occupe une place de choix dans les assolements fourragers intensifs de l'Ouest de la France. Aussi, avons-nous jugé impératif d'aborder ce type d'étude quand nos travaux nous ont amené à nous intéresser au fonctionnement des systèmes fourragers intensifs de la Bretagne occidentale, systèmes principalement à base de maïs et de ray-grass d'Italie. En effet, de nombreux enseignements peuvent être attendus des études à caractère écophysiologique : meilleure compré- hension du fonctionnement des cultures, valorisation des facteurs de production, adaptabilité des espèces... etc., autant de connaissances qui permettent de mieux maîtriser la culture, d'accroître la régularité de sa production, donc la sécurité de l'agriculteur.

Bien que le ray-grass d'Italie soit une plante réputée d'installation facile, il n'en reste pas moins que l'implantation de celle-ci peut être contrariée par divers facteurs :

- un semis à une époque peu favorable. C'est souvent le cas dans l'Ouest de la France où l'intensification des systèmes de cultures fourragères conduit à mettre en place cette plante très tôt, en fin d'hiver, ou très tard, en fin d'automne (par exemple après une culture de maïs récolté tardivement) ; 
- diverses agressions lors des premiers stades : adventices (souvent très abondantes lors de la $1^{\text {re }}$ coupe, ce qui diminue considérablement la qualité du fourrage), parasites animaux (oscinies, taupins, lapins...) et parasites végétaux (champignons appartenant à divers genres: Fusarium, Pythium, Septoria).

Il importe donc de mieux maîtriser l'implantation de la culture, ce qui peut être rendu plus facile par une bonne connaissance des facteurs du milieu actif sur les premières phases du cycle de la plante, et, en particulier, sur la phase semis-levée.

Divers facteurs interviennent sur la réalisation du stade «levée »d'une plante : des facteurs intrinsèques (réserves du grain, faculté et énergie germinative...), des facteurs extrinsèques dont deux types sont généralement retenus : les facteurs culturaux, en particulier la préparation du lit de semences qui influe sur le degré d'oxygénation des semences (DASHBERG \& MENDEL, 1971 ; COME, 1975) ; les facteurs du milieu, d'origine climatique, dont deux sont généralement cités par divers auteurs : la température et l'humidité du lit de semences (BLACKLOW, 1974 $a$ et $b$; BUCKLE, 1972 ; FredDES, 1972; Hough, 1972; SingH \& DHALIWAL, $1972 \ldots)$. Dans cette $1^{\text {re }}$ partie, nous ne nous intéresserons qu'à l'influence du facteur température, les conditions expérimentales étant telles que les autres facteurs déterminants sur cette phase sont considérés comme non limitants.

\section{II. - MATÉRIEL ET TECHNIQUE EXPÉRIMENTALE}

\section{A) La plante}

La variété de ray-grass d'Italie choisie est « Tiara », qui est l'une des variétés les plus largement répandues en Bretagne occidentale. Les semences sont issues de récoltes récentes et de bonne faculté germinative.

\section{B) Les boîtes de germination}

Les semences sont placées entre deux papiers filtres, à l'intérieur d'une demi-boîte de Petri. Un vase sans bec, rempli d'eau et renversé sur les deux papiers filtres, assure leur humidification permanente (fig. 1).

Vingt semences individualisées sont disposées dans chaque boîte. Ces dernières sont placées dans des enceintes obscures thermostatées, la gamme de températures couvertes allant de 0 à $35^{\circ} \mathrm{C}$.

La température est mesurée à l'aide d'un thermomètre mouillé, placé au-dessus de la boîte de Petri. Sa température

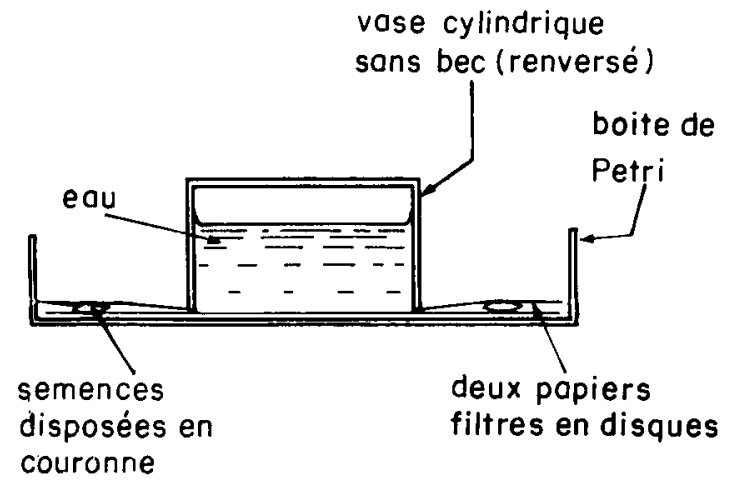

Figure 1

Boîte de germination.

Germination box. est proche de celle du papier filtre humide, donc de celles des graines en germination ou des jeunes plantules. Les relevés sont effectués 3 fois par jour. Les amplitudes thermiques observées sont faibles, puisqu'elles ne dépassent pas $3{ }^{\circ} \mathrm{C}$.

On peut remarquer que ce n'est pas la température de l'enceinte qui est mesurée. En effet, un contrôle précis de l'humidité relative a montré que celle-ci variait de 40 à 100 p. 100 selon les températures recherchées. Or la différence entre la température du papier filtre (thermomètre humide) et celle de l'enceinte (thermomètre sec) est d'autant plus grande que l'humidité relative est faible. La mesure de la température au niveau de l'enceinte conduirait à une erreur relativement importante d'estimation de la température : par exemple, pour une humidité relative de 50 p. 100 , nous avons pu observer une température de l'enceinte de $35^{\circ} \mathrm{C}$, alors que la température du papier filtre n'était que de $26,6{ }^{\circ} \mathrm{C}$, soit une différence de $8,4^{\circ} \mathrm{C}$.

\section{C) Mesures et observations}

Les mesures et observations effectuées sont illustrées par la figure 2 qui décrit l'évolution de la plantule de ray-grass d'Italie du semis à la levée.

\section{SEMIS GERMINATION}
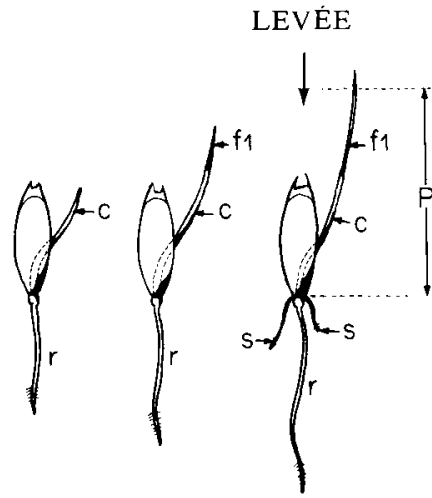

Figure 2

Evolution de la plantule du semis à la levée. ( $r$ : radicule: $c$ : coléoptile; $f 1$ : première feuille; $s$ : racines séminales latérales; $P$ : profondeur du semis).

Plantule evolution from sowing to emergence.

$(r:$ radicle $; c:$ shoot $; f 1:$ first leaf $; s:$ first lateral roots $; P:$ depth of sowing).

Sont mesurés: l'augmentation de poids des semences lors de la phase d'imbibition en eau (par pesées horaires, puis journalières), l'accroissement en longueur de la radicule, du coléoptile puis de la $1^{\text {re }}$ feuille (par mesures journalières).

Sont comptées les apparitions : des radicules (germination), des racines séminales latérales, des premières feuilles lors de la percée du coléoptile par celles-ci. Rappelons que, par convention, un stade phénologique est atteint quand 50 p. 100 des plantules vivantes présentent le caractère considéré. En ce qui concerne le stade "levée ", nous ne pouvons, dans cette expérience, que le simuler ; il s'agit donc d'un stade théorique, atteint quand la longueur du coléoptile (ou de la $1^{\text {re }}$ feuille mesurée depuis la base du coléoptile) est égale ou supérieure à la profondeur supposée du semis. Les coléoptiles étant mesurés étirés, les vitesses de levée obtenues par cette méthode seront plus élevées que celles observées avec un semis en place. Dans ce cas, du fait de la position aléatoire de la semence dans le sol, les coléoptiles présentent des courbures les plus variées, ce qui retarde leur émergence. 
croissance visible. L'élongation du coléoptile n'est pas encore observable, par transparence, à travers les enveloppes. L'incidence de la température sur la croissance de la radicule est présentée figure 6.

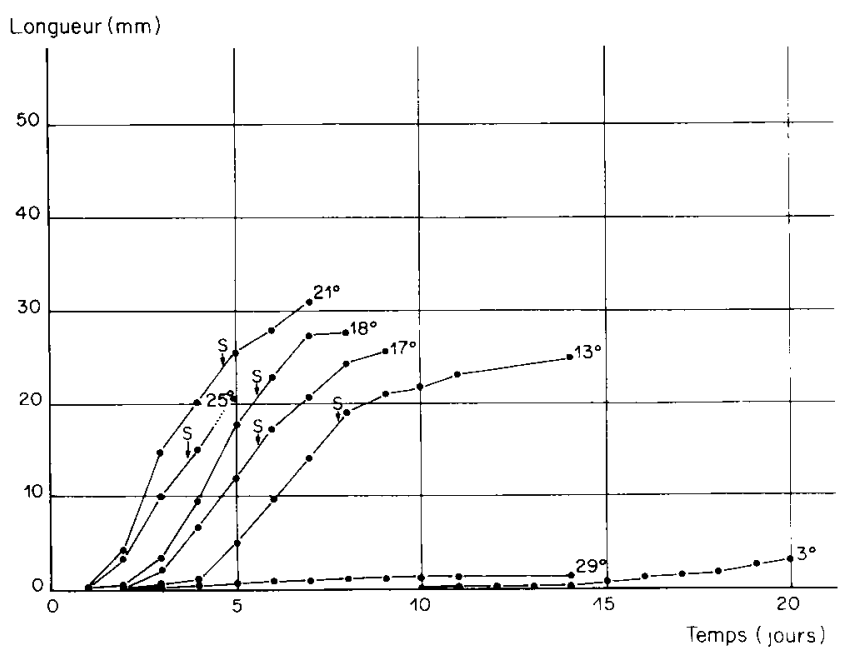

Figure 6

Courbes de croissance de la radicule pour différentes températures ( $S$ indique le moment où $50 \mathrm{p} .100$ des systèmes radiculaires séminaux présentent les premières racines latérales).

Growth curves of the radicle for several temperatures ( $S$ " points out the moment where $50 \mathrm{p} .100$ of the seminal root systems show the first lateral roots).

L'élongation de la radicule est pratiquement nulle pour une température de $3^{\circ} \mathrm{C}$. Celle-ci est d'autant plus rapide que la température augmente, jusqu'à un optimum proche de $21^{\circ} \mathrm{C}$. Au-delà, la croissance se ralentit pour tendre vers zéro au voisinage de $29^{\circ} \mathrm{C}$. On peut remarquer un classement régulier des températures croissantes jusqu'à $21^{\circ} \mathrm{C}$ puis décroissantes jusqu'à $29^{\circ} \mathrm{C}$.

L'apparition des premières racines séminales latérales se produit quand la longueur de la radicule est de l'ordre de $20 \mathrm{~mm}$.

L'effet dépressif des températures basses ou élevées sur la croissance de la radicule n'est pas du même type. Pour les températures élevées, la croissance de la radicule se bloque rapidement (alors que le coléoptile continue parfois sa croissance), des nécroses apparaissent sur la jeune racine, ce qui entraîne la mort de certaines plantules en quelques jours. Le taux de germination étant déjà diminué pour ces températures, peu de plantules survivent après une quinzaine de jours. Pour les températures basses, par contre, la croissance apparaît normale mais lente. Après un mois d'observation, toutes les plantules sont encore vivantes.

\section{D) Influence de la température sur la croissance du coléoptile puis de la première feuille}

L'influence de la température sur la croissance du coléoptile relayé ensuite par la $1^{\text {re }}$ feuille est comparable à celle observée sur le système radiculaire (fig. 7). A $3{ }^{\circ} \mathrm{C}$, la croissance est faible mais régulière. Entre 21 et $25^{\circ} \mathrm{C}$, la croissance est optimale. A $29^{\circ} \mathrm{C}$, après une croissance légère, le coléoptile se décolore, devient plus ou moins translucide et meurt ; après une quinzaine de jours, toutes les plantules sont mortes. La longueur maximale atteinte par le coléoptile semble indépendante de la température. La $1^{\text {re }}$ feuille perce le coléoptile quand celui-ci mesure de 10 à $15 \mathrm{~mm}$ de longueur.

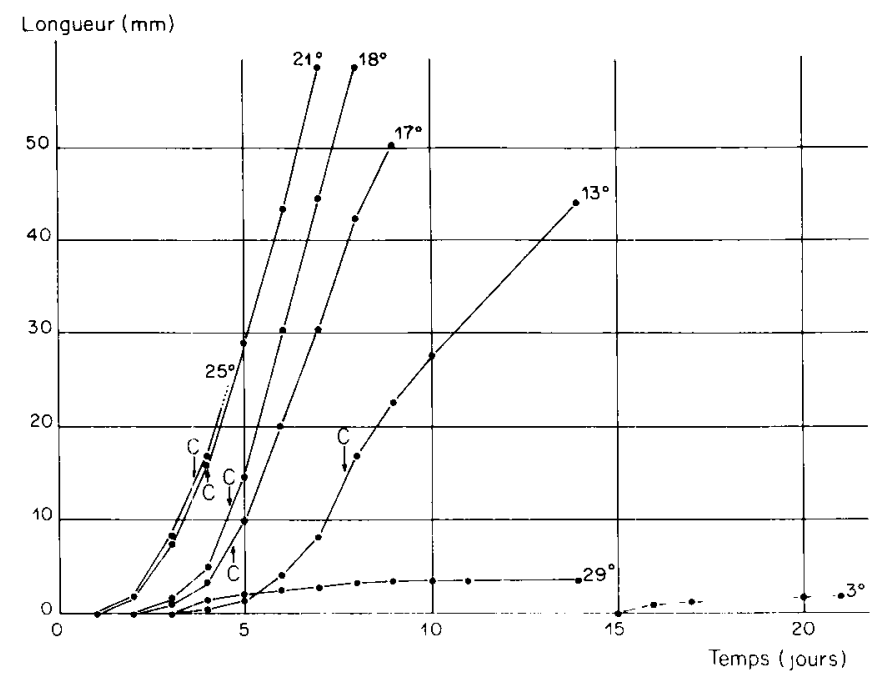

Figure 7

Courbes de croissance du coléoptile (relayé par la $1^{r e}$ feuille) pour différentes températures. ( $C$ indique le moment où $50 \mathrm{p} .100$ des premières feuilles percent les coléoptiles).

Growth curves of the shoot (relieved by the first leaf) for several temperatures. ( $C$ » points out the moment where 50 p. 100 of the first leaves sprout).

\section{E) Influence de la température sur la phase semis-levée}

Les stades "levée " mesurés ici sont des stades théoriques considérés comme atteints quand 50 p. 100 des coléoptiles (ou des premières feuilles mesurées depuis la base du coléoptile) présentent une longueur supérieure ou égale à la profondeur du semis. Sur la figure 8 , nous avons présenté les vitesses de levée, ainsi obtenues, en fonction de la température. Trois profondeurs de semis, couvrant les valeurs obtenues dans la pratique agricole sont exposées.

L'allure générale de ces courbes est voisine de celle des vitesses de germination. Elles en diffèrent légèrement par les valeurs critiques : le seuil thermique inférieur est voisin de $1{ }^{\circ} \mathrm{C}$ (au lieu de $0{ }^{\circ} \mathrm{C}$ ), le seuil thermique supérieur est de l'ordre de $29^{\circ} \mathrm{C}$ (au lieu de $32^{\circ} \mathrm{C}$ ). A $29^{\circ} \mathrm{C}$, en effet, la germination est possible, mais les plantules ne peuvent survivre, la levée ne peut, de ce fait, se produire. L'optimum thermique est du même ordre de grandeur : 20 à $25^{\circ} \mathrm{C}$.

\section{F) Caractérisation climatique de la phase semis-levée}

La figure 8 montre que, en dessous de l'optimum thermique, la relation entre la vitesse de levée et la température, est de type linéaire. En ne prenant en compte que les points situés en dessous de $25^{\circ} \mathrm{C}$, on peut remarquer que les nuages de points correspondant aux différentes profondeurs de semis s'ajustent de façon satisfaisante à des droites (tabl. 1).

La relation entre vitesse de levée $1 / \mathrm{N}$ (ou V) et température est donc du type, $V=a T-b=a\left(T-\frac{b}{a}\right)$ relation qui peut aussi s'écrire

$$
\mathrm{V}=\mathrm{a}\left(\mathrm{T}-\mathrm{T}_{0}\right)
$$

Il apparaît ici que le seuil thermique $\mathrm{T}_{0}$ est voisin de $1{ }^{\circ} \mathrm{C}$ $(1,09$ à 1,64$)$.

C'est sur cette linéarité de relation que repose l'hypothèse des sommes de températures (DURAND, 1969). En se plaçant dans les conditions climatiques océaniques de la Bretagne 


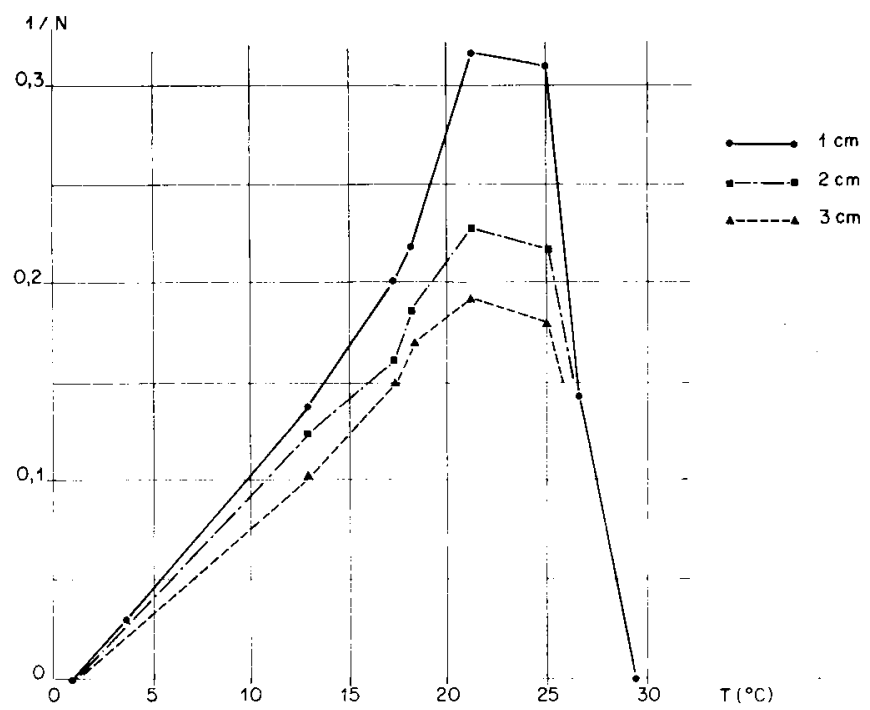

Figure 8

Vitesse de levée en fonction de la température, pour trois profondeurs de semis : 1,2 et $3 \mathrm{~cm}$ ( $N$ est la durée de la phase semis-levée).

Speed of emergence in relationship with temperature, for three depths of sowing : 1,2 and $3 \mathrm{~cm}(N=$ length in days from sowing to emergence).

occidentale, donc pour les valeurs de températures correspondant à celles de la gamme envisagée ci-dessus, on peut penser que la méthode des sommes de températures peut s'avérer intéressante pour caractériser la phase semis-levée du ray-grass d'Italie en conditions naturelles, en l'absence, bien sûr, d'autres facteurs limitants. Un calcul rapide montre que la somme thermique nécessaire pour accomplir la phase semis-levée serait, selon la profondeur de semis, de : $71^{\circ} \mathrm{J}$ (pour $1 \mathrm{~cm}$ ), $91^{\circ} \mathrm{J}$ (pour $2 \mathrm{~cm}$ ) et $111^{\circ} \mathrm{J}$ (pour $3 \mathrm{~cm})$.

\section{IV. - CONCLUSION}

Cette étude effectuée en conditions contrôlées montre le rôle déterminant de la température sur les différentes phases de croissance et de développement qui conduisent à la levée du ray-grass d'Italie. Cette action se traduit par : une augmentation de la vitesse d'imbibition des semences avec les températures croissantes (du moins, jusqu'à $29^{\circ} \mathrm{C}$ ), une variation en cloche des vitesses de germination. de croissance du coléoptile puis de la $1^{\text {re }}$ feuille et de la radicule, et des vitesses de levée. Un certain nombre de valeurs repères de la température ont pu être déterminées avec précision : zéro de germination $\left(0^{\circ} \mathrm{C}\right)$, zéro de levée $\left(1^{\circ} \mathrm{C}\right)$, optimum de germination et de levée (environ $\left.20-25^{\circ} \mathrm{C}\right)$, seuil supérieur de germination $\left(32^{\circ} \mathrm{C}\right)$, seuil supérieur de levée $\left(29^{\circ} \mathrm{C}\right)$.

Le rôle de la température sur la phase semis-levée du ray-grass d'Italie étant maintenant précisé, il reste à analyser l'action de ce facteur en présence d'autres facteurs limitants, en particulier le facteur hydrique. C'est ce que nous proposons de faire dans une $2^{\mathrm{e}}$ partie où la phase semis-levée est étudiée en conditions naturelles.

Reçu le 19 septembre 1980 Accepté le 23 janvier 1981

TABLEAU 1

Relation entre vitesse de levée $(1 / N)$ et température $(T)$ pour trois profondeurs théoriques de semis (pour des températures inférieures à $25^{\circ} \mathrm{C}$ ) Relationship between daily rate of emergence $(1 / \mathrm{N})$ and temperature $(T)$ for three theorical depths of sowing (for temperatures lower than $\left.25^{\circ} \mathrm{C}\right)$

\begin{tabular}{|c|c|c|c|c|c|}
\hline $\begin{array}{l}\text { Profondeur } \\
\text { du semis } \\
(\mathrm{cm})\end{array}$ & $\begin{array}{c}\text { Relation entre } 1 / \mathrm{N} \\
\text { et } \mathrm{T}\left({ }^{\circ} \mathrm{C}\right)\end{array}$ & $\begin{array}{l}\text { Coefficient de } \\
\text { détermination } \\
\qquad\left(R^{2}\right)\end{array}$ & $\begin{array}{l}\text { Coefficient de } \\
\text { corrélation } \\
\text { (R) }\end{array}$ & d.d.l. & $\begin{array}{l}\text { Niveau de } \\
\text { signification }\end{array}$ \\
\hline 1 & $\begin{array}{c}1 / \mathrm{N}=0,014 \mathrm{~T}-0,023 \\
\text { ou } \\
1 / \mathrm{N}=0,014(\mathrm{~T}-1,64)\end{array}$ & 0,960 & 0,980 & 4 & $1 \%$ \\
\hline 2 & $\begin{aligned} 1 / \mathrm{N} & =0,011 \mathrm{~T}-0,012 \\
\text { ou } & \\
1 / \mathrm{N} & =0,011(\mathrm{~T}-1,09)\end{aligned}$ & 0,988 & 0,995 & 3 & $1 \%$ \\
\hline 3 & $\begin{array}{c}1 / \mathrm{N}=0,009 \mathrm{~T}-0,011 \\
\text { ou } \\
1 / \mathrm{N}=0,009(\mathrm{~T}-1,22)\end{array}$ & 0,991 & 0,996 & 3 & $1 \%$ \\
\hline
\end{tabular}

\section{RÉFERENCES BIBLIOGRAPHIQUES}

Blacklow W. M., 1974a. Influence of temperature on germination and elongation of the radicle and shoot of corn. Guelph Proj., Final Rep., 280-287.

Blacklow W. M., 1974b. Simulation model to predict germination and emergence of corn in an environment of changing temperature. Crop Sci. 13 (6), 604-608.

Buckle J. A., 1972. Temperature effect on maize emergence. Rhodesian Agric. J. 69 (5), 90.

Come D., 1975a. Quelques problèmes de terminologie concernant les semences et leur germination. In La germination des semences. Gauthier-Villars, 11-26.

Come D., 1975b. Rôle de l'eau, de l'oxygène et de la température dans la germination. In La germination des semences. GauthierVillars, 27-44.
Dashberg S., Mendel K., 1971. The effect of soil water and aeration on seed germination. J. Exp. Bot. 22 (73), 992-998.

Durand R., 1969. Signification et portée des sommes de températures. Bull. Techn. Inform. 238, 185-190.

Freddes R. A., 1972. Effect of water and heat on seedling emergence. J. Hydrol. 16, 341-359.

Hough M. N., 1972. Weather factors affecting the development of maize from sowing to flowering. J. Agric. Sci. Camb. 78, 235-331.

Livingstone R. E., 1916. Physiological temperatures indices for the study of plant growth in relation to climatic conditions. Physiol. Res. 1 (8), 399-420.

Singh N. T., Dhaliwal G. S., 1972. Effect of soil temperature on seedling emergence in different crops. Plant Soil 37 (2), 441-444. 